\title{
UN TEXTO ETNOGRÁFICO EN TZELTAL DE SIBACÁ
}

\author{
Por Evangelina Arana O. \\ Instituto Nacional de Antropología \\ e Historia.
}

Sibacá es una pequeña localidad indígena del Estado de Chiapas situada al $\mathrm{SW}^{F}$ de Ocosingo, cabecera del municipio del mismo nombre. Los habitantes de Sibacá llegan a 800 y en un elevado porcentaje son monolingües, es decir, no hablan español, sino sólo su lengua materna que es el tzeltal. Los bilingües son hombres principalmente, que han salido a trabajar en las fincas cafetaleras, como lo hacen gran parte de los indígenas de la región, tzeltales y tzotziles, mediante una contratación francamente desventajosa.

Los hombres cultivan en sus tierras frijol y maíz; este último se da en abundancia, por lo que no sólo obtienen para su consumo familiar; sino que pueden vender ese grano que es básico en su alimentación, a los pueblos indígenas de las tierras frías que sufren gran escasez en los meses de julio y agosto. Las mujeres se dedican a los quehaceres domésticos, hacen ollas, cazuelas y comales de barro, tejen fajas, todo para su uso familiar y crían gallinas, guajalotes, patos y puercos. Huevos y aves los venden en Ocosingo generalmente y los cerdos en pie a los compradores que llegan de San Cristóbal de las Casas; en esta forma ayudan a aumentar los ingresos de la familia. Asimismo, participan en las cosechas y se contratan por días para recoger café en las fincas cercanas, pero siempre regresan diariamente a su hogar.

Estuvimos en Sibacá durante los meses de diciembre de 1959 a enero de 1960, con el objeto de estudiar el idioma. Tratamos de recoger leyendas o cuentos de la región; pero ninguno de los informantes sabía historias; unas cuantas personas dieron anécdotas personales, pero la mayor parte no sabía de qué platicar. Formulamos una serie de preguntas en tzeltal, de tipo personal y etnográfico. Las llamamos "censo" por ser una pa- 
labra conocida por la gente y que contesta sin problema. Este artificio permitió a cada uno comenzar a hablar; aun cuando sólo contestaban a las preguntas específicas, nos daban ejemplos de expresiones y vocabulario, y a la vez proporcionaban datos etnográficos interesantes. Por otra parte, muchas veces inspiró al informante a contar una que otra experiencia personal. Ciertas preguntas especialmente tuvieron este efecto, las relacionadas con los familiares, las enfermedades, el trabajo y los viajes.

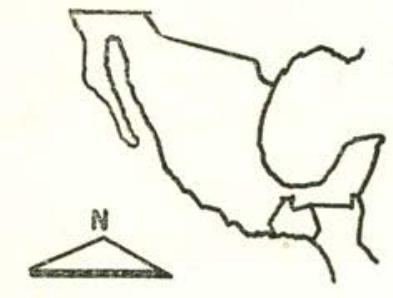

- BACHAJON

- OCOSINGO

SIBACA

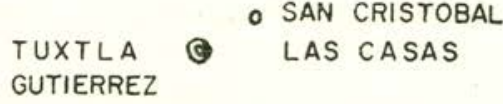

COMITAN

Localización de Sibacá respecto a otras poblaciones de Chiapas.

El texto que se analiza es uno de los varios que pudimos obtener para el análisis del idioma. Sirve como muestra del habla de este pueblo. En general, cada pueblo tzeltal tiene sus modalidades lingüísticas aunque todos se entienden entre sí. El idioma de Sibacá se asemeja bastante al de Bachajón que se localiza al norte, según puede verse en el mapa. Ambos pueblos sostienen diversas relaciones de orden económico y social.

El texto abunda en préstamos del español, como: obligación, alférez, capitán, porque trata de instituciones que tienen su origen en la primera época colonial. Menciona las obligaciones de tipo religioso o civil que un hombre debe cubrir para obtener respeto y prestigio en su sociedad. 


\section{TeXto EN IDIOMa CON SU TRAdUCCión analítica}

Te mero primero kaj-on ta a?te-l, entonse pati-l El mero primero empezar-yo en trabajo, entonces después bo-on ta a?te-l ta karretera. i te tareya wake-?b metro, ir-yo en trabajo en carretera. Y la tarea $6 \quad$ metros i te s-toyo-l-il te ch?en ay oxe-b? metro. I te a?te-l, y el su-altura la cueva ser 3 metros. Y el trabajo te karretera, jun semana, wake-?b k?ajk?a-l, i te a?te-l la carretera, una semana, 6 días, y el trabajo obligasyon nax ma-?y-uk s-tojo-l. i lego bo-on ta obligación sólo, no tener su-pago. Y luego ir-yo en san kristobal y-u?un ba-k-ich? be tale-l y-ijkatz San Cristóbal su-causa ir-yo-traer para él venida yo-carga te albanil y-u?un ta s-jajch jun ch?ul-naj. el albañil su-causa en él-levantar una sagrada-casa. i pati-l jajch te ixkweledo ta okosingo i bwen $\mathrm{Y}$ después comenzar la escuela en Ocosingo y bien ajch?-em te ji?. Bweno, obligasyon, ma-?y-uk. mojar-hecho la arena. Bueno, obligación no-tener s-tojo-l. Bweno, i la-j-kuch bwen k?ajk? ta te su-pago. Bueno, y pas-yo cargar bien caliente en la j-pat i ma-?y-uk s-tojo-l. i pati-l bo-on ta mi-espalda y no-tener su-pago. Y después ir-yo en saltore?awa $y$-u?un laj-k-ich?-bae-l te partira y-u?un Salto-de-agua su-causa pas-yo-coger-ida la partida su-causa te chitam. Tey e la-j-pas ganar ja?-nax los puercos. De allí pas-yo-hacer ganar eso-sólo lajune-?b y-ox-winik $\quad \mathrm{i}$ te kastiya sinkwenta. Bweno i diez su-tercer-veinte y en castilla cincuenta. Bueno y 
$\begin{array}{lllll}\text { pati-l } & \mathrm{k} \text { ?alal mero } & \text { lek-ix } & \mathrm{k}-\mathrm{o} \text { ?tan } & \text { bo-on } \\ \text { después } & \text { cuando mero } & \text { bien ya } & \text { mi-corazón } & \text { ir-yo }\end{array}$

laj-k-ich?-tal che-?b kreso koeton y-u?un pas-yo-coger-venir dos gruesas cohetones su-causa ch?ul-naj i pati-l, la-j-pas ta obligasyon sagrada-casa y después pas-yo-hacer en obligación y-u?un te iglesya. Bweno, ta primero, och te k-a?te-l su-causa la iglesia. Bueno, en primero, entrar el mi-trabajo s-bi-i-l alperes-il y-u?un la-j-pas $\quad y$-u?un su-nombre alférez su-causa pas-yo-hacer su-causa ta ch?ul-naj. ta s-che-?b-a-l welta, och-on ta kapitan en sagrada-casa. En su-segunda vez, entrar-yo en capitán y-u?un te ch?ul iglesya i la-j-pas yan welta su-causa la sagrada iglesia y pas-yo-hacer otra vez kapitan $y-u$ ? un te ch?ul iglesya, pero ma?-to capitán su-causa la sagrada iglesia, pero no-exacto jich-uk te bin ti la-j-pas. Ja?-nax puro trawu así lo que pas-yo-hacer. Eso-sólo puro trago la-s-k?an te pobre k-ermano-tik, ja? te pas-ellos-querer los pobres hermano-pl., ese lo namey ja? me?-to entonse la-j-pas te karnabal i antiguo eso así-exacto entonces pas-yo-hacer el carnaval y sok ?oxe-?b limeton puru trawu, y-u?un $\mathrm{k}$ ? ax junto 3 garrafones puro trago. su-causa pasar te karnabal. la-j-lajin lajun-winik peso te $\mathrm{k}$ ? ax te el carnaval. Pas-yo-acabar diez-veintes pesos el pasar el karnabal. I och-on ta mayordomo ta s-kwenta y-u?un carnaval. Y entrar-yo en mayordomo en su-cuenta su-causa j-tat-ik jesukristo i la-j-man jun kreso koeton nuestro-padre J. y pas-yo-comprar una gruesa cohetones 
$\mathrm{k}$ ?alal $\mathrm{k}$ ? ax te s-k?in te j-tat-ik jesukristo. cuando llega su-fiesta el nuestro-padre Jesucristo.

Ora yo?tik ya-x-ba-j-pas ch?e-?b xan welta

Ora ahora pres-ir-yo-hacer dos más vuelta

mayordomo y-u?un j-tat-ik san markus, i sok mayordomo su-causa nuestro-padre San Marcos, y junto

y-u? j-ala-me?-tik natibira ya-to-j-pas

su-causa nuestra-extensa-madre natividad pas-todavía-yo-hacer

te s-k?ina-l ya-to-j-pas-bey

la su-fiesta, pres-todavía-yo hacer te s-k?in.

la su-fiesta. ya-to-j-pas

pres-todavía-yo-hacer

tz?aka-l te serbi-l y-u?un te iglesya i te k?alal acabado el servicio su-causa la iglesia y el cuando pres-yo-descansar-ya mi-corazón. Como acabado-ya su-causa

te s-manta-l te ch?ul iglesya. I laj-ix k-o?tan. el su-mandato la sagrada iglesia. Y acabar-ya mi-corazón.

\section{TraduCción LiBre}

La primera vez que trabajé fue en la carretera. La tarea era de 6 metros planos de una altura de 3 metros. Ese trabajo duró una semana, seis días; pero no recibía pago sino era sólo obligación. Luego fui a San Cristóbal a buscar un albañil para que levantara la sagrada casa (iglesia). Después ayudé a levantar la escuela de Ocosingo; cargaba arena que estaba bien mojada. Era obligación, no recibía pago. Bueno, se ponía caliente mi espalda de tanto cargar; pero no tenía pago. Después fui a Salto de Agua para llevar una partida de puercos. Gané cincuenta pesos. Bueno, y después ya tranquilo mi corazón fui, compré y regresé con 2 gruesas de cohetones para la sagrada 
casa (iglesia). Entré después al servicio de la iglesia; primero hice mi trabajo de alférez en la sagrada casa (iglesia). En la segunda vez entré de capitán a la sagrada iglesia, hice otra vez más de capitán pero no como lo hacían los pobres hermanos que sólo beber querían, así era antiguamente. Entonces hice el carnaval y junté 3 garrafones de trago para pasar el carnaval, gasté doscientos pesos para pasar el carnaval. Entré de mayordomo de Nuestro Señor Jesucristo. Compré una gruesa de cohetones para pasar la fiesta de Nuestro Señor Jesucristo.

Ahora hago de mayordomo, por segunda vez, de Nuestro Padre San Marcos y haré otra vez más para Nuestra Señora de la Natividad, todavía voy a hacer su fiesta. Con esta vez más acabaré mi servicio para la iglesia y entonces descansa ya mi corazón. Acabaré como lo manda la sagrada iglesia. Terminé ya de hablar ("Acabó ya mi corazón").

Observaciones: El texto se ha transcrito usando símbolos, en lo posible, con el mismo valor que en el español y separando con guiones las distintas partes componentes de cada palabra. La oclusión glotal se marca con ?, equivale al conocido "saltillo" en la lingüística mexicana y consiste en un cierre abrupto de las cuerdas vocales. No se escribe aquí el referido fonema cuando se encuentra al principio de la palabra, ya que es implícito en todo caso en que se encuentre una vocal escrita al principio de la palabra. Este signo se emplea entre y después de consonantes, y además siguiendo a algunas oclusivas que se glotalizan en el tzeltal como t?, ch?, tz? k?

La x se usa para el sonido que tenía el español antiguo en "baxo" bajo, semejante a la ch del francés y la sch del alemán.

La w es la u consonante, parecida a gu del español.

\section{Préstamos del español}

\section{a) Particulas}

Los préstamos incluyen no solamente nombres sino verbos, partículas, elementos de todas clases. En la primera línea tenemos "mero primero", palabras que en español son adjetivos, pero que en el tzeltal funcionan como partículas. Cada una de ellas se puede usar independientemente, "primero" para decir 
al principio, por primera vez; "mero" como un elemento enfático.

Más adelante se encuentran las palabras "entonse", "puru", "komu". La primera se usa sin s final; las dos últimas han cambiado la o final en $\mathrm{u}$, como sucede, por lo general, con todos los préstamos del español que tienen esta misma característica. Puede advertirse que hay fluctuación, según se observa en el mismo texto en aquellas personas que tratan de hablar mejor el español, al que llaman "castilla".

\section{b) Nombres}

Los nombres que aquí figuran como préstamos es común que se adapten a la fonética tzeltal, como "tareya", "metro" y "peso" tarea, metros, pesos ( $\sin$ la s del plural porque no es el sufijo que usan con ese valor en el idioma nativo), "albanil" albañil (el sonido ñ es ajeno al tzeltal); "ixkweledo", "saltore?áwa", "partira", "natibira", respectivamente escuelero (los mestizos de la región llaman así a los niños que asisten a la escuela); Salto de Agua, nombre de una población de la zona; partida. En estas palabras se sustituye por $\mathrm{r}$, la d que no existe en tzeltal, pero este sonido se comienza a introducir y en ixkweledo hasta tenemos, como ultracorrección, d en lugar de r. Luego, en "kreso" gruesa se sustituye g por k; en "alperes-il" alférez, la $f$ por $\mathrm{p}$ y además añaden el sufijo -il en este caso da la idea del cargo; "k-ermano-tik" mis hermanos según la fonética nativa y los afijos, el posesivo $\mathrm{k}$ - $m i$ y -tik plural; "limeton" garrafón del español antiguo limeta equivalente a botella. En la zona equivale a $3 / 4$ de litro. "s-kwenta" su cuenta, la s- inicial indica su de él o ella.

"S-manta-l" su manda(r), s- inicial es de su y con la terminación de los sustantivos del tzeltal.

"Serbi-l" servi(r), ajustado como en el caso anterior.

\section{c) Verbos}

Como ejemplo de verbos prestados del español tenemos "ganar" que es de uso común en el sentido de ganar dinero por un trabajo. 


\section{Combinaciones TZELTAL - ESPAÑOL}

Es común en tzeltal juntar la palabra española con su equivalente en tzeltal tal vez para hacer énfasis.

También encontramos "ora yo?tik" ora, ahora, la primera aféresis de la segunda. De igual modo se usa "kwando k?alal", "para y-u?un", "i sok" expresiones en que la palabra tzeltal es más o menos equivalente. En la expresión "ch?ul iglesya" sagrada iglesia, tenemos una frase mixta esencialmente igual a "ch?ul naj" sagrada casa o iglesia.

\section{ANOTACiONES SOBRE EL TZELTAL}

"te" se usa antes de los sustantivos que no son poseídos y tiene el valor de los artículos el o la y sus plurales.

Los calificadores se anteponen al nombre como en "ch?ul naj" sagrada casa, "porbre k-ermano-tik" mis pobres hermanos (pobre mi-hermano-pl.), "tut alal" chiquito niño "yax te?" verde árbol, o ceiba, "muk?u-l chay" gran pez.

Los posesivos se anteponen a los sustantivos, ejemplos: "s- bi?il" su nombre, "K-a?tel" mi trabajo, "j-tat-ik" nuestro padre. Tenemos: K- y j- mi iniciales según principie la palabra por vocal o consonantes; aw- o a-, $t u$; y- o s- para $s u$ en las mismas condiciones que para $m i$. Estas letras añadiendo al final la terminación -tik, se usan para los plurales.

Los anteriores prefijos y terminaciones se usan con los verbos transitivos para indicar las personas que ejecutan la acción: yo, tú, él o ella, etc. Ejemplo: "ya-j-pas", yo hago. La partícula "ya" o "yax" se antepone a los verbos para indicar el tiempo presente, seguida del sujeto y la raíz verbal. El pasado se indica iniciando la forma verbal con la partícula "laj" o "la". Ejemplo: "la-j-kuch" cargué, "la-j-pas" hice, "laj-k-il" vi.

Los verbos intransitivos sólo agregan después de la raíz verbal las terminaciones "-on", "-at" y la $3^{n}$ persona no lleva terminación, para indicar el sujeto de la acción en el singular. En el plural añaden "-otik" (reducción de "-on-tik"), "-ex", "-ik". Ejemplo: "bo-on" voy, literalmente ir-yo, "och-on" entro, entrar-yo. Los mismos sufijos pueden emplearse con sustantivos, como "muk?-at" eres grande, "s-tat-on" soy su papá (su-papá-yo). 
Una interesante construcción verbal muy común en la lengua tzeltal se encuentra en expresiones como: "laj-k-ich?-tal" lo traje, literalmente pasado-yo-coger-venida. Se trata de compuestos verbales, que abarcan dos verbos, como este ejemplo, o hasta tres. En conjunto expresan un movimiento, una acción realizada y el regreso al punto de partida. Puede incluirse dentro de esa construcción compleja el objeto directo o indirecto, como en: "ba-k-ich?-be talel y-ijkatz" ir-yo-coger-para él venida su-carga, o sea fui y le traje su carga.

En cuanto a la numeración podemos decir que tiene como base un sistema vigesimal y actualmente sólo llega a 400 en tzeltal. Del 1 al 10 cada número tiene su nombre; del 11 al 19 son composiciones a base de diez, así 12 es "laj-cha?ye?b" diez-dos, 15 "jo?-lajune?b" cinco-diez. El 20 es "ta?b" pero para contar las veintenas se usa "winik" que es hombre. Ejemplo: "lajun-winik" diez veintes, 200; "lajune?b y-ox--winik" diez su tres veinte, o sea diez de la tercera veintena, equivalente a 50. En este pueblo hoy día usan generalmente los numerales nativos hasta 100 y las centenas las dicen en español, menos 400 que es "bajk?", un zonte. 
Una interesante construcción verbal muy común en la lengua tzeltal se encuentra en expresiones como: "laj-k-ich?-tal" lo traje, literalmente pasado-yo-coger-venida. Se trata de compuestos verbales, que abarcan dos verbos, como este ejemplo, o hasta tres. En conjunto expresan un movimiento, una acción realizada y el regreso al punto de partida. Puede incluirse dentro de esa construcción compleja el objeto directo o indirecto, como en: "ba-k-ich?-be talel y-ijkatz" ir-yo-coger-para él venida su-carga, o sea fui y le traje su carga.

En cuanto a la numeración podemos decir que tiene como base un sistema vigesimal y actualmente sólo llega a 400 en tzeltal. Del 1 al 10 cada número tiene su nombre; del 11 al 19 son composiciones a base de diez, así 12 es "laj-cha?ye?b" diez-dos, 15 "jo?-lajune?b" cinco-diez. El 20 es "ta?b" pero para contar las veintenas se usa "winik" que es hombre. Ejemplo: "lajun-winik" diez veintes, 200; "lajune?b y-ox--winik" diez su tres veinte, o sea diez de la tercera veintena, equivalente a 50. En este pueblo hoy día usan generalmente los numerales nativos hasta 100 y las centenas las dicen en español, menos 400 que es "bajk?", un zonte. 